\title{
Fertilizantes de entrega controlada: potencial para mejorar la productividad de la reforestación
}

\author{
Controlled-release fertilizers: potential for enhanced reforestation productivity \\ ROBIN ROSE ${ }^{1}$, DIANE L. HAASE ${ }^{1}$, EDUARDO ARELLANO² \\ ${ }^{1}$ Department of Forest Science, Oregon State University, 321 Richardson Hall, Corvallis, OR, USA, 97331. \\ E-mail: robin.rose@orst.edu; diane.haase@ orst.edu \\ ${ }^{2}$ Departamento de Ciencias Forestales, Pontificia Universidad Católica de Chile, Casilla 305, \\ Correo 22, Santiago, Chile. E-mail: earellan@puc.cl
}

\begin{abstract}
SUMMARY
Controlled-release fertilizers (CRFs) have a great potential to increase forest seedling growth and competitiveness at a variety of outplanting sites. However, CRFs are often poorly understood. In order to use them successfully, their formulation, release behavior, and environmental interactions must be considered. This synthesis paper describes types of CRF (organic, nitrogen-reaction products, and coated products) and their associated research findings. The advantages and disadvantages associated with each CRF are also reviewed, and some tips for their usage are offered. Some current research challenges in further understanding the best ways to use CRF in forestry applications are listed.
\end{abstract}

Key words: seedling nutrition, fertilization, nursery production, coated fertilizers.

\section{RESUMEN}

Los fertilizantes de entrega controlada (FEC) presentan un gran potencial para aumentar la competitividad de las plantas forestales para una variedad de sitios de reforestación. Sin embargo, normalmente existe un pobre conocimiento sobre ellos. Con el fin de usar los FEC en forma exitosa se deben considerar su formulación, el patrón de entrega y sus interacciones ambientales. Este trabajo sintetiza los distintos tipos de FEC (orgánicos, productos con nitrógeno de lenta solubilidad y productos recubiertos) y diversas investigaciones asociadas a su utilización. Además, revisa las ventajas y desventajas asociadas a los FEC, se realizan ciertas recomendaciones sobre su utilización y menciona algunos de los desafíos para futuras investigaciones destinadas a mejorar la comprensión en las formas de aplicar los FEC en situaciones forestales.

Palabras clave: nutrición de plántulas, fertilización, producción en vivero, fertilizantes recubiertos.

\section{INTRODUCCION}

Los fertilizantes de entrega controlada (FEC) han sido utilizados en la horticultura por décadas. Los FEC no han sido solamente aplicados en invernaderos y producción en contenedores, sino que también en diversas operaciones agrícolas, cam- pos de golf, huertos, paisajismo y producción de sustratos. En los últimos años estos fertilizantes han ido ganando popularidad en la fertilización forestal. En aplicaciones directas, realizadas al momento de plantar en la zona radicular, se demostró que los FEC estimulaban el crecimiento en pino oregón (Pseudotsuga menziesii (Mirb.) Fran- 
co) 1-0 plug $(1,2)$ y en plántulas de segunda temporada de abeto occidental (Tsuga heterophylla Raf. Sarg.) (3). Krasowski et al. (4) encontraron que los FEC estimulaban el desarrollo radicular y el estado nutricional de picea blanca (Picea glauca [Moench] Voss).

Mientras esta tecnología presenta un gran potencial para mejorar la eficiencia en el uso de fertilizantes, el crecimiento y la competitividad de plantas forestales, aún se requiere analizar cuidadosamente otras características propias de estos productos. Al momento de establecer un régimen nutricional que supla las demandas nutricionales de las plántulas en determinados estados de desarrollo, resulta clave entender las diversas formulaciones, forma de entrega de los nutrientes y su interacción con el medio ambiente.

El objetivo de este artículo es (1) describir las características de los FEC (2), entregar información sobre las diferencias entre los productos (3), revisar algunas referencias encontradas en la literatura, y (4), entregar recomendaciones generales sobre el uso de estos fertilizantes en forma efectiva, sin recomendar ningún producto en particular. La intención es entregar información útil sobre la utilización de FEC que puede beneficiar a viveristas, productores de plantas y personas vinculadas a la reforestación.

FERTILIZANTES DE ENTREGA CONTROLADA. El término "entrega-controlada" (también referido a "entrega o descarga lenta") es usado para describir fertilizantes que no liberan el $100 \%$ de los nutrientes disponibles al momento de la aplicación. La entrega de los nutrientes es variable según el producto y depende de los niveles de humedad del suelo, la temperatura y/o la actividad microbiológica. Existen tres categorías básicas: orgánicos, nitrógeno de solubilidad lentas y los recubiertos. El objetivo final de los FEC es entregar nutrientes a una tasa que coincida con la demanda de la planta y de esa forma evitar pérdidas. Esto trae como consecuencia una máxima eficiencia en su utilización al optimizar la calidad de la planta, disminuyendo la frecuencia en la fertilización y las pérdidas por lixiviación. Sin embargo, existen límites en el nivel de control sobre la entrega, los cuales se discutirán a continuación.

PRODUCTOS ORGANICOS. La utilización de productos orgánicos como fuente de fertilizantes es una actividad cultural realizada durante miles de años. Estos incluyen guanos de animales, desechos de pescado, algas marinas, lombrices, huesos, aserrín y desechos de plantas compostadas (5). Estos productos suelen presentar un menor porcentaje de nutrientes en relación a los fertilizantes sintéticos $\mathrm{y}$, por lo mismo, deben ser aplicados en mayor cantidad. Los elementos provenientes de estos fertilizantes pueden ser muy variables y resulta muy difícil predecir su patrón de entrega. Sin embargo, estos productos han sido utilizados en una gran variedad de situaciones, resultando en una alternativa económica a la utilización de FEC.

En un estudio con tres variedades de pino (Pinus spp.) se utilizaron desechos orgánicos secos como fertilizantes, produciéndose un incremento en los niveles de biomasa en comparación a plántulas fertilizadas con una fuente inorgánica o no fertilizadas (6). En otro estudio, se detectaron deficiencias de magnesio en plantaciones de abeto grande americano (Abies grandis (Dougl. ex D. Don) Lindl.) y pino oregón fertilizadas con residuos orgánicos. Sin embargo, no se encontró ningún tipo de otra deficiencia o toxicidad nutricional, y las carencias fueron corregidas con aplicaciones de $\mathrm{MgSO}_{4}$ (7). Weetman et al. (8) encontraron incrementos en la concentración de nutrientes en abeto occidental, pinabeto del Pacífico (Abies amabilis (Dougl.) Forbes), y tuya gigante (Thuja plicata Don ex D. Don) tratadas con desechos orgánicos. Aplicaciones de compost de desechos de pescado mejoraron el crecimiento y los niveles de concentración foliar de plantaciones cloróticas de tuya gigante (9).

PRODUCTOS A BASE DE NITROGENO DE BAJA SOLUBILIDAD. Los productos a base de nitrógeno (N) son creados por intermedio de reacciones químicas de un componente nitrogenado soluble en agua y aldehídos. Esto resulta en una estructura molecular compleja con niveles de solubilidad limitados. Una vez introducidos a la solución del suelo, estos productos se liberan y transforman lentamente en formas químicas disponibles para la planta.

UREA-FORM. Urea-form es uno de los grupos que se obtienen de la reacción de urea y formaldhehído. Estos productos están compuestos por polímeros de monometilol-urea de diverso tamaño y solubilidad. Cadenas de polímeros más extensas pre- 
sentan niveles de solubilidad menor en comparación a cadenas más cortas, en períodos más extensos. Urea-form es la clase menos soluble y contiene al menos un $35 \%$ de $\mathrm{N}$ total, del cual al menos un $15 \%$ es urea no reactiva (10). Urea-form es un sólido blanco inoloro y su descomposición está regulada principalmente por la actividad microbiológica en el suelo (11). Por lo tanto, la liberación de nutrientes dependerá de factores ambientales que regulan la actividad microbiológica en el suelo (temperatura del suelo, humedad, pH y aireación). Dentro de los productos comerciales que contienen urea-form se encuentran los pellets de Agriform ${ }^{\circledR}$ (O.M. Scotts \& Sons, Marysville, OH) y Nitroform ${ }^{\circledR}$ (Nu-Gro Technologies, Inc., subsidiaria de Nu-Gro America Corporation, Brantford, Ontario, Canada). Las formas más solubles de ureas metilenizadas se pueden encontrar en fertilizantes de otras compañías como Scotts (O.M. Scotts \& Sons) y Nutralene ${ }^{\circledR}$ (Nu-Gro Technologies, Inc.).

Las urea-form han sido utilizadas en forma exitosa como un fuente de $\mathrm{N}$ controlado. Austin y Strand (12) encontraron efectos positivos en el crecimiento de plántulas después de aplicar ureaform. En un estudio que buscaba examinar la mineralización, nitrificación y lixiviación de $\mathrm{N}$ en un suelo forestal ácido, Nitroform ${ }^{\circledR}$ produjo un incremento en la disponibilidad de $\mathrm{N}$ mineral sin aumentar los niveles de nitrificación y disminuyendo los riesgos de lixiviación de $\mathrm{NO}_{3}$ (13). Gleason et al. (14) aplicaron saquitos de urea-form $\left(\right.$ Agriform ${ }^{\circledR}$ ) a plantas de pino ponderosa (Pinus ponderosa Dougl. ex Laws) y encontraron un efecto positivo en el crecimiento al finalizar la segunda y tercera temporada de crecimiento en un sitio de pobre disponibilidad inicial de nutrientes. En un estudio con plántulas de acacia de tres espinas (Gleditsia triacanthos L.), se determinó que al finalizar la segunda temporada de crecimiento los diámetros fueron mayores cuando recibieron Nitroform ${ }^{\circledR}$ en relación al tratamiento no fertilizado (15).

Otra investigación realizada para comparar el efecto de distintos FEC en plántulas de pino oregón en diversos suelos arcillosos y condiciones de humedad, se encontró que las plántulas fertilizadas con Wilgro ${ }^{\circledR}$ (mezcla de Nitroform con fuentes solubles de nutrientes distintos al $\mathrm{N}$ ) presentaron una mejor respuesta en diámetro, altura y desarrollo radicular, especialmente bajo tratamiento de alto contenido de humedad en el suelo. En condiciones de estrés hídrico, ninguno de los fertilizantes utilizados mostró un efecto significativo sobre las plántulas (16) (figura 1).

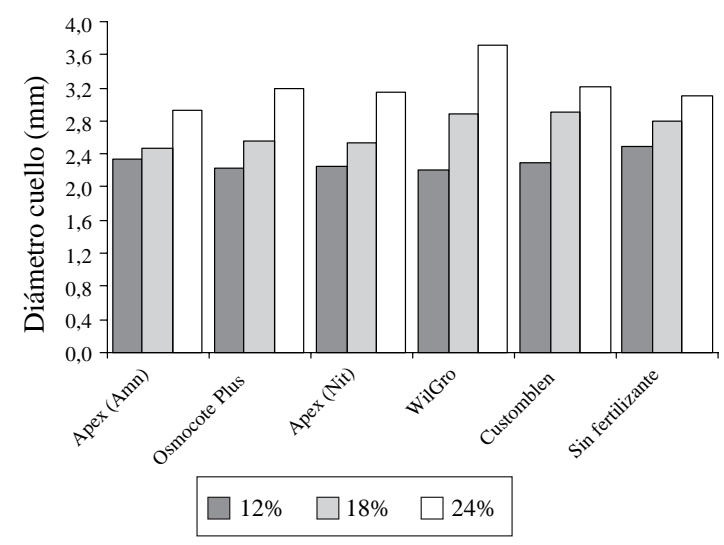

Figura 1. Valor promedio estimado del diámetro del cuello para plántulas de pino oregón fertilizadas con cinco tipos de FEC y tres niveles de contenido de humedad en el suelo, después de una temporada de crecimiento (16).

Mean seedling stem diameter for seedlings grown with five fertilizer treatments, and three soil moisture contents, after one growing season (16).

IBDU. Las Isobutilidendiurea (IBDU), como las urea-form, se crean a partir de la reacción de urea y aldehídos, y tienen una solubilidad en agua menor a un $0,1 \%$. Los fertilizantes comercializados contienen cerca de un $30 \%$ de $\mathrm{N}$ (17). Los IBDU son sólidos, blancos, cristalinos, disponibles tanto en partículas finas como gruesas (10). A diferencia de las urea-form, el $\mathrm{N}$ en los compuestos IBDU se torna disponible para la planta a través de la hidrólisis. La tasa de entrega del nutriente dependerá entonces del tamaño de los gránulos de IBDU y el contenido de agua en el suelo. Este proceso de liberación se puede acelerar en condiciones de acidez y altas temperaturas (10). Los IBDU son manufacturados por Nu-Gro Technologies, Inc. y se encuentran disponibles en concentrados para mezclar con fertilizantes o en saquitos.

Se encontró que IBDU en saquitos, aplicados en combinación con fertilizantes solubles, produjeron plantas más largas de roble americano (Quercus rubra L.) y túpelo (Nyssa sylvatica Marsh.), en comparación a los tratamientos que recibieron los fertilizantes solubles o de IBDU por 
separado (18). Rose y Ketchum (19) utilizaron saquitos de IBDU de N:P:K a razón 14:3:3 o 9:9:4 al momento de plantación de pino oregón, pino ponderosa, abeto occidental y sequoia (Sequoia sempervirens (D. Don) Endl.), encontrando un efecto significativo sobre el tamaño de las plántulas en dos de cinco sitios con contenido de humedad del suelo adecuado. Por otro lado, Nambiar y Cellier (20) encontraron nula respuesta a la aplicación de IBDU en plántulas de pino radiata (Pinus radiata D. Don).

PRODUCTOS RECUBIERTOS. A diferencia de los productos nitrogenados de baja solubilidad, los fertilizantes recubiertos permiten la liberación controlada de otros nutrientes además del N. Los FEC recubiertos consisten en un concentrado de nutrientes soluble recubierto por una capa insoluble al agua. Diversos materiales para recubrir los fertilizantes han sido utilizados incluyendo ceras, aceites, azufre, plásticos y resinas. Las más comunes son las cubiertas polimeradas. Un polímero es un compuesto de alto peso molecular derivado de la concentración de moléculas más pequeñas. Para situaciones de fertilizantes de corta duración es recomendable una cubierta delgada y liviana. Sin embargo, para períodos de nutrición más largos, mezclas de entrega a corto o largo plazo o mezclas de cubiertas delgadas y gruesas son recomendables para entregas más balanceadas (21).

UREA RECUBIERTA CON AZUFRE. LOS fertilizantes recubiertos con azufre fueron una de las primeras tecnologías de fertilización controladas disponibles. A veces, una capa de cera microcristalina es utilizada como aislante. Las ureas recubiertas con azufre (SCU) liberan nutrientes a través de la penetración del agua en las fallas, poros e imperfecciones de la película que cubre el fertilizante. Una vez que el agua ha penetrado, la urea disuelta es rápidamente liberada desde el gránulo (10). La tasa de entrega se encuentra controlada por el grosor y calidad de la cubierta del fertilizante.

En un estudio que buscaba analizar el efecto de la fertilización con urea de un rodal de 24 años de abeto occidental, se encontró que el área basal y el crecimiento en volumen por árbol aumentó significativamente en los tratamientos que recibieron SCU en relación a los no fertilizados o a los fertilizados sólo con urea (22).
FERTILIZANTES RECUBIERTOS CON POLIMEROS. LOS FEC recubiertos con polímeros son considerados los de mayor avance tecnológico debido a su alta eficiencia en el control de la entrega de nutrientes y la duración del producto. En la mayoría de los sistemas horticulturales estos fertilizantes han reemplazado a los productos recubiertos con azufre debido a la entrega gradual y consistente de nutrientes que los recubiertos con polímeros poseen (10). Los FEC basados en una cubierta polimerizada se pueden clasificar en resinas termoset o resinas termoplásticas. La entrega de nutrientes de estos fertilizantes depende tanto del grosor y composición de la cubierta como de las características ambientales del sustrato donde es aplicado.

La información sobre la tasa de duración de estos fertilizantes se obtiene donde los propios manufacturadores y normalmente va entre un período de 3 a 18 meses. Sin embargo, es importante resaltar que esta información es determinada en condiciones controladas de temperatura en laboratorio (aprox. $21^{\circ} \mathrm{C}$ ). La entrega real bajo condiciones de terreno puede ser considerablemente variable entre distintos productos y normalmente a una tasa bastante más desuniforme. La mayoría de los fertilizantes descargan un porcentaje considerable de nutrientes inmediatamente después de su aplicación. La entrega de lo remanente dependerá del tiempo, temperatura y la humedad del suelo (23, 24). El mecanismo de entrega ocurre en dos etapas (25). En la primera, la presión de vapor se infiltra en los gránulos y se condensa en las sales de fertilizante soluble, aumentando la presión osmótica dentro del gránulo. En una segunda etapa, la presión dentro del fertilizante trae como consecuencia el goteo lento de la solución con fertilizante hacia la solución del suelo. En la medida que la solución dentro del gránulo se va diluyendo, la presión y la liberación de fertilizantes disminuyen. Kochba et al. (26) examinaron la entrega de nutrientes a diversas temperaturas, encontrando que el paso determinante en la liberación de nutrientes desde estos fertilizantes es la migración del vapor de agua desde el suelo hacia el grano de fertilizante.

La variabilidad en la descarga de los FEC recubiertos con polímeros puede ser afectada por la abrasión de la cubierta (27) y por almacenaje. Los gránulos de fertilizantes almacenados están sujetos a recibir el impacto de la humedad, tem- 
peratura y de la propia manipulación. Los fertilizantes almacenados, especialmente en contenedores abiertos, pueden comenzar a hidratarse y liberarán nutrientes en forma más rápida en cuanto sean colocados en el medio de crecimiento (28).

NUTRICOTE $^{\circledR}$. Este producto es elaborado por ChissoAsahi Fertilizer Company (Tokio, Japón). La cubierta es una resina termoplástica altamente impermeable. En este caso se incorporan agentes de control de liberación como el etileno-vinil acetato y diversos surfactantes (10). La cantidad presente en la cubierta del fertilizante determinará la tasa de entrega. En este caso la descarga está controlada principalmente por la temperatura del suelo.

Plántulas de abeto occidental que fueron fertilizadas con $50 \mathrm{~g}$ Nutricote ${ }^{\circledR}$ al momento de plantación ('tipo 360', 16-10-10, 12-meses de duración a $25^{\circ} \mathrm{C}$ ) fueron significativamente más largas al tercer año, para los diversos grupos fertilizados en comparación a las plántulas que no recibieron fertilizante (29). Reddell et al. (30) incorporaron dos productos Nutricote ${ }^{\circledR}$ ('Blue', 4-5 meses de duración, y 'Black', 6-9 meses de duración, ambos con una formulación 16-4.4-8.3) en el medio de crecimiento de dos especies tropicales (Gmelina arborea Roxb. y Acacia mangium Willd.) a tasas desde 0 a $20 \mathrm{~g}$ de fertilizante $\mathrm{x}^{-1}$. En las plantas que recibieron los niveles de fertilización más altos, los incrementos en índice de volumen fueron superiores al 100\%. Esto contrastó con la ausencia de cualquier tipo de efecto en el crecimiento de los árboles a tasas de fertilización mucho más altas realizadas en experimentos previos, usando el mismo tipo de suelo, pero fertilizantes solubles. Los autores concluyen que la localización del fertilizante en las cercanías de las raíces de los árboles establecidos incrementó la habilidad de éstos para competir con las malezas, probablemente debido a una alta eficiencia en el uso de nutrientes para favorecer el crecimiento de los árboles.

OSMOCOTE $^{\circledR}$. Este producto, manufacturado por O.M. Scotts Company (antiguamente Grace Sierra Horticultural Products Company), ha sido utilizado por décadas en diversos sectores silvoagropecuarios. La tecnología de cubierta de resina involucra un fertilizante central soluble, cubierto por un copolímero termosensible de diciclopentadieno y un éster-glicerol disuelto en un solvente alifático de hidrocarbono (10). En el mercado, existe una serie de fertilizantes Osmocote ${ }^{\circledR}$ con una gran variabilidad en las tasas de entrega.

De todos los productos presentes en este trabajo, Osmocote ${ }^{\circledR}$ es el FEC de aplicación más común. Brand y Janas (31) encontraron que $30 \mathrm{~g}$ de Osmocote ${ }^{\circledR}$ (17-6-10) mejoraron la disponibilidad de nutrientes después de la plantación en pino blanco occidental (Pinus monticola Dougl. ex D. Don) $\mathrm{y}$ picea blanca. Bajo buenas condiciones de humedad, plántulas de abeto de Sitka (Picea sitchensis (Bong.) Carr.) fertilizadas con Osmocote ${ }^{\circledR}$ mostraron diferencias significativas en crecimiento comparadas con las plántulas no fertilizadas. Sin embargo, en condiciones de estrés de humedad, las plántulas fertilizadas sólo mostraron una leve diferencia en crecimiento promedio (32). Walker (33) encontró que la utilización de Osmocote ${ }^{\circledR}(17-$ 7-12) en plántulas de pino amarillo (Pinus jeffreyi Grev. \& Balv.) al momento de plantar estimulaba el crecimiento y disminuía la mortalidad, especialmente en aplicaciones de $20 \mathrm{~g}$ por planta. Sin embargo, otros dos FEC utilizados en este estudio $\left(\right.$ Sierra ${ }^{\circledR} 16-6-10+$ Minors $^{\circledR}$ y High $\mathrm{N}^{\circledR}$ 24-4-7) mostraron un pobre efecto en el crecimiento, y una mortalidad significativa en plántulas que recibieron una alta dosis de fertilizantes, especialmente de High $\mathrm{N}^{\circledR}$ 24-4-7. Plántulas de vivero de Engelmann spruce (Picea engelmannii Parry ex Engelm.) fertilizadas con Osmocote ${ }^{\circledR}$ o con Nutricote ${ }^{\circledR}$ tenían un menor peso radicular, pero un mayor largo de tallo, diámetro de cuello y peso total que los tratamientos control que sólo recibieron fertilizante soluble (34). Walker y Huntt (35) aplicaron FEC a plántulas de pino amarillo producidas en contenedores (Sierra 17-6-12 y Agriform ${ }^{\circledR}$ 14-4-6 tabletas). Después de 12 meses, los volúmenes verdes de tallo y raíces promedio de las plántulas fertilizadas fueron un $100 \%$ mayores a las plántulas no fertilizadas.

Productores de pino radiata y eucaliptos (Eucalyptus spp.) de Brasil, Chile y Argentina han incorporado aplicaciones de Osmocote Miniprill ${ }^{\circledR}$ al medio de crecimiento (18-5-9 con tres meses de duración para pino y 19-6-10 con cuatro meses de duración para eucaliptos), con resultados exitosos en dosis cercanas a los $3,5 \mathrm{~kg}$ por $\mathrm{m}^{3}$ de sustrato.

El efecto de Osmocote ${ }^{\circledR}$ no sólo ha sido evaluado a nivel de crecimiento de las plántulas. Castellano et al. (36) y Hunt (34) demostraron que aplicaciones de Osmocote ${ }^{\circledR}$ reducen la formación de micorrizas en plantas en contenedores, mien- 
tras que Chakravarty y Chatarpaul (37) encontraron que el desarrollo de ectomicorrizas de tamarak (Larix laricina (Du Roi) K. Koch) fue sustancial, sin importar la aplicación de Osmocote ${ }^{\circledR}$ (17-610) y/o un fertilizante soluble (10-52-10). Shaw et al. (38) encontraron que la colonización de hongos tiende a aumentar con aplicaciones más bajas de FEC, pero las plántulas de abeto de Sitka fueron más grandes a tasas de fertilización mayores.

POLYON ${ }^{\circledR}$. Este fertilizante corresponde al nombre genérico de fertilizantes recubiertos con poliuretano, distribuidos por Simplot bajo el nombre de Apex ${ }^{\circledR}$ (Pursell Technologies, Inc.). La liberación del fertilizante ocurre por difusión osmótica y se encuentra controlada por el grosor de la capa que cubre el fertilizante. Este producto está creado a partir de capas reactivas en las cuales los monómeros son polimerizados a medida que entran en contacto con el sustrato (10). Se reconoce a los fertilizantes Polyon ${ }^{\circledR}$ como los menos reactivos a temperaturas extremas porque el poliuretano se mantiene rígido, intacto y no se expande.

En un estudio en plantas producidas en contenedores y fertilizadas con Apex ${ }^{\circledR}$, el volumen del tallo se incrementó en cerca de un $70 \%$ después de cuatro temporadas de crecimiento en terreno en relación a plantas no fertilizadas con FEC (39) (figura 2). En otro estudio, plántulas de pino oregón recibieron una variedad de tratamiento de humedad y de fertilización con productos de entrega controlada. Después de una temporada de crecimiento, las plántulas fertilizadas con Osmocote ${ }^{\circledR}$ y Apex ${ }^{\circledR}$ no presentaron diferencias significativas en el volumen radicular y de tallo, en relación a las plántulas fertilizadas con mezclas de FEC con fertilizantes solubles y las no fertilizadas, independiente de los niveles de humedad del suelo (16) (figura 3).
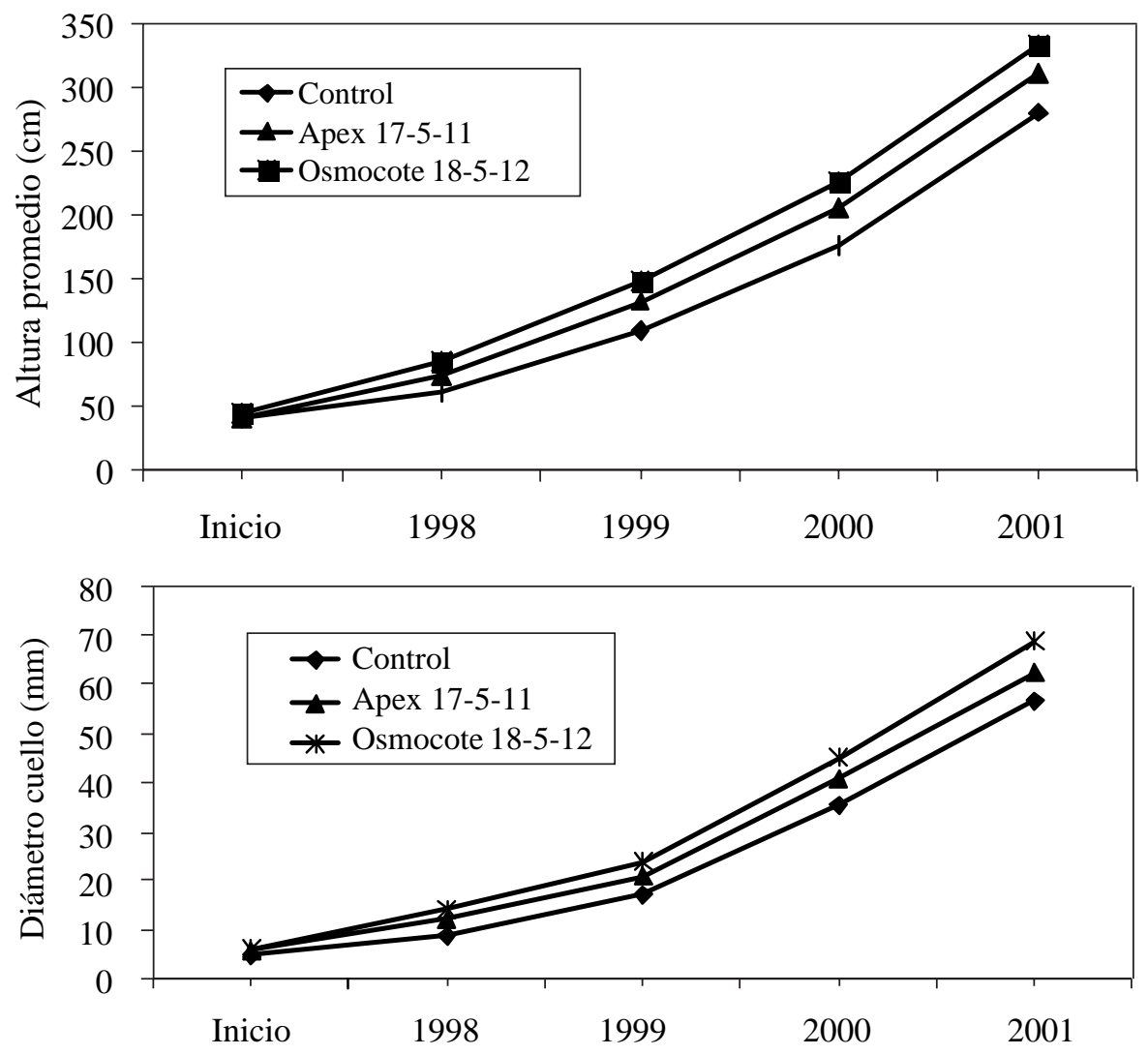

Figura 2. Cuarta temporada de crecimiento después del establecimiento; la altura y el diámetro promedio fueron mayores para las plántulas que recibieron FEC en el vivero (39). Four growing seasons after outplanting. Mean height and stem diameter (greatest for nursery-grown container seedlings in CRF-amended media) (39). 


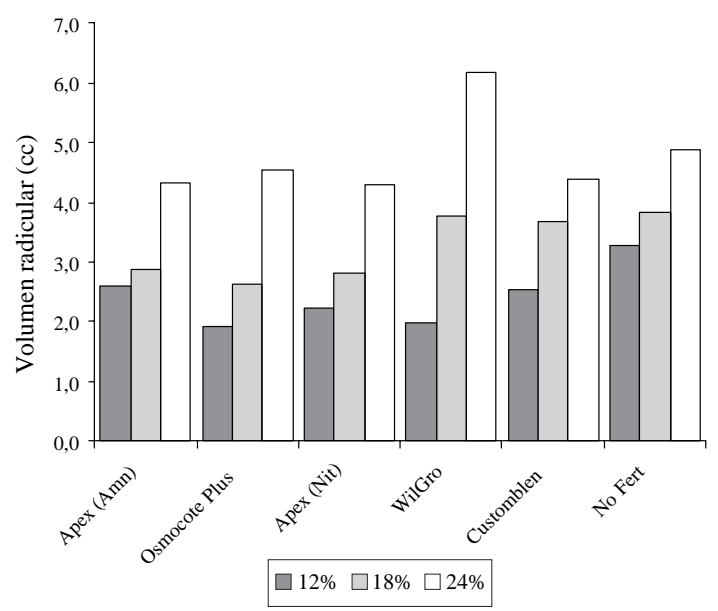

Figura 3. Valor promedio estimado del volumen radicular para plántulas de pino oregón fertilizadas con cinco variedades distintas de FEC y tres niveles de humedad en el suelo, después de una temporada de crecimiento (16).

Mean seedling root volumes after one growing season for seedlings grown with five fertilizer treatments and three soil moisture contents (16).

VENTAJAS Y DESVENTAJAS. Al momento de ser analizados como una clase de fertilizantes, los FEC tienen ventajas y desventajas específicas. La comprensión clara de estas características permite optimizar la aplicación operacional de este tipo de productos. Los FEC no serán siempre recomendables, ya sea para viveros o aplicaciones en terreno.

- Ventajas. La principal y más clara ventaja de los FEC sobre los fertilizantes solubles de entrega rápida es su habilidad de suministrar nutrientes a las plantas por períodos de tiempo prolongado sólo mediante una aplicación. Debido a su lenta descarga, las posibilidades de daños a las plantas asociadas a niveles de toxicidad se reducen y la eficiencia en el uso de los fertilizantes mejora ostensiblemente. Otra ventaja es la clara disminución en los niveles de pérdidas de nutrientes por lixiviación $(40,41)$. Comúnmente, se piensa que los FEC son costosos en comparación a los fertilizantes solubles en agua. Sin embargo, las constantes aplicaciones requeridas cuando se usan estos últimos pueden resultar más costosas en relación a los FEC (21).

Los FEC se pueden utilizar en una gran variedad de situaciones, ya sea en invernaderos, viveros a raíz desnuda y fertilizaciones en terreno.
Walker y Huntt $(42,43)$ encontraron que, después de una selección cuidadosa de las formas de nutrientes y tasas de aplicación, los FEC son un buen complemento en la producción de plántulas con características requeridas para sitios de reforestación específicos.

- Desventajas. La principal desventaja en la utilización de FEC se encuentra en la posibilidad de que ocurran entregas de nutrientes descontroladas. A diferencia de los productos solubles, los FEC no pueden ser ajustados una vez que han sido aplicados. Al momento de usar las formulaciones Osmocote ${ }^{\circledR}$ (3-4 meses) y Nutricote ${ }^{\circledR}$ en plantas en maceteros, durante las primeras dos semanas las descargas más rápidas de $\mathrm{N}$ y $\mathrm{K}$ disminuyeron las demandas de las plantas por nutrientes, incrementando las pérdidas por lixiviación (44). En otro estudio se encontró que para 17 tipos distintos de FEC existía una entrega desigual de nutrientes, con tasas de liberación más altas en las primeras semanas, especialmente en tratamientos con mayores temperaturas y con períodos de entrega de nutrientes más cortos (45). En estos casos, el suministro de nutrientes puede no corresponder a los requerimientos de las plantas, por lo que las pérdidas por lixiviación pueden aumentar; incluso dentro de productos recubiertos con polímeros similares la intensidad y el patrón de entrega pueden ser variables (46). Huett y Gogel (45) encontraron que para fertilizantes Apex ${ }^{\circledR}$ los niveles de entrega de nutrientes podían ser hasta 10 semanas superiores a productos de etiquetado similar pertenecientes a Osmocote ${ }^{\circledR}$.

Además, el patrón de entrega puede variar de acuerdo al tipo de nutrientes. Se determinó que para Nutricote ${ }^{\circledR}$ y Osmocote ${ }^{\circledR}$ la liberación de fósforo $(\mathrm{P})$ puede ser sólo entre un 60 a $80 \%$ del $\mathrm{N}$ y potasio (K) entregado (47). Huett y Gogel (45) encontraron una tendencia similar entre diversos FEC como Nutricote ${ }^{\circledR}$, Osmocote ${ }^{\circledR}$ y Apex $^{\circledR}$ en relación a la entrega de $\mathrm{P}$, siendo siempre más lenta en relación al $\mathrm{K}$, que al mismo tiempo fue más lento que el N. Alzugaray (48) demostró que después de 14 meses, sólo entre un $19 \%$ y $38 \%$ del P había sido liberado, mientras que el $\mathrm{K}$ y el $\mathrm{N}$ lo hicieron siempre a tasas mayores (entre $48 \mathrm{y}$ $79 \%$ y entre 63 y $85 \%$, respectivamente) (figura 4). Esto puede traer como consecuencia una pobre relación $\mathrm{N}: \mathrm{P}$ e inadecuados niveles de $\mathrm{P}$ para el desarrollo de la planta. 


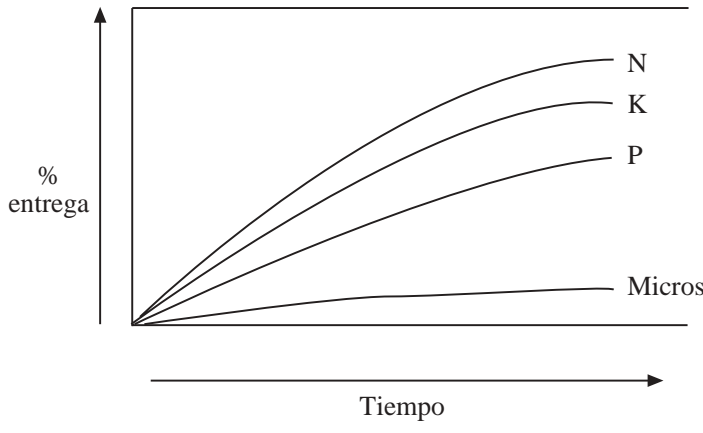

Figura 4. La entrega real de $\mathrm{N}$ y $\mathrm{P}$ difiere en forma significativa para determinar tasas de entrega en terreno (Alzugaray 2002).

Differing release of $\mathrm{N}$ and $\mathrm{P}$ in a field trial to assess $\mathrm{CRF}$ release rates (Alzugaray 2002).

CONSIDERACIONES AMBIENTALES. La magnitud en la respuesta de las plantas a la aplicación de FEC es notablemente distinta de sitio a sitio. Factores propios del suelo, especialmente el contenido de humedad, pueden tener un profundo efecto en el nivel de respuesta a la fertilización. En un estudio con FEC del tipo Apex ${ }^{\circledR}$ y Osmocote ${ }^{\circledR}$ en plántulas de pino oregón, las respuestas en diámetro al quinto año fueron significativamente diferentes entre las establecidas en un sitio costero y en un sitio seco del interior (39). En un estudio de 10 años en fertilización de pino ponderosa en tres sitios diferentes, las respuestas a la fertilización fueron siempre mejores en la situación de menor estrés hídrico de la planta (49). La competencia con malezas, la fertilidad del suelo y las condiciones de sitio también afectan el tipo de respuesta a la aplicación de FEC. La falta de control de competencia puede restringir la adecuada respuesta a la fertilización $(50,51)$.

La temperatura es otro factor que afecta fuertemente el comportamiento de los FEC. Las tasas de entrega de $\mathrm{N}, \mathrm{P}$ y $\mathrm{K}$ en diversos fertilizantes: Osmocote ${ }^{\circledR}$, Apex $^{\circledR}$ y Nutricote ${ }^{\circledR}$, aumentan entre un $13 \%$ y un $19 \%$ con un aumento de temperatura de $10{ }^{\circ} \mathrm{C}$ (44). Kochba et al. (26) encontraron que en aumentos de $10{ }^{\circ} \mathrm{C}$ en la temperatura del suelo, la liberación de nutrientes en fertilizantes polimerizados aumentaba en el doble. La posible "caída" de los fertilizantes bajo altas temperaturas puede traer como consecuencia una acumulación tóxica de sales, especialmente bajo sitios con altos niveles de estrés hídrico. En un vivero, esto necesariamente se lixiviará con agua. En terreno, esto puede producir un pobre crecimiento e incrementar la mortalidad (figura 5).

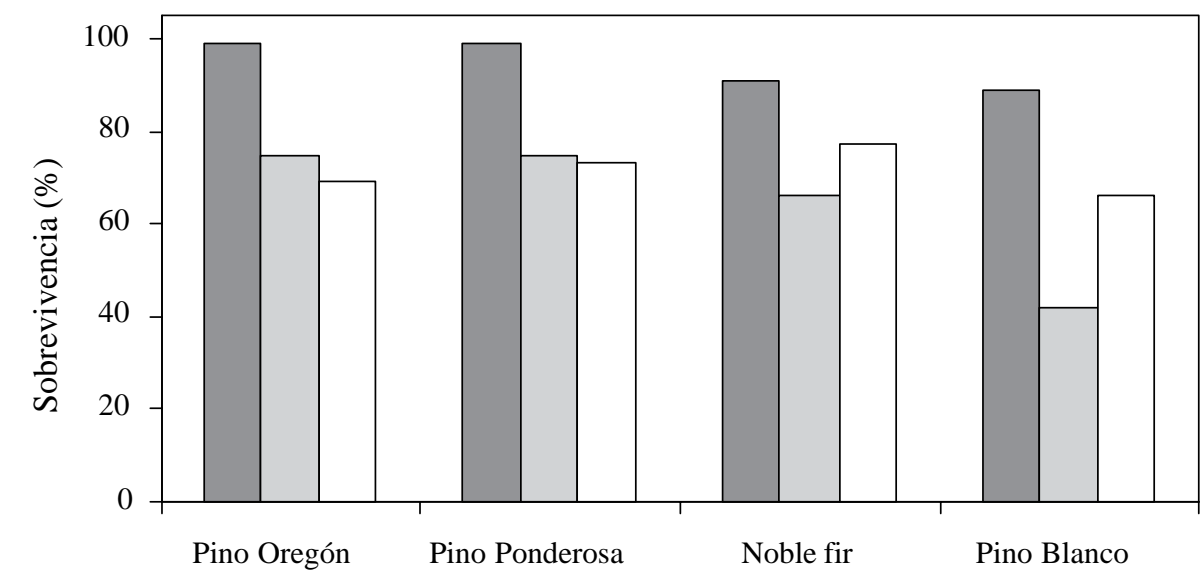

$\square$ Control $\square$ Osmocote 15-9-12 $\square$ Forestcote 22-4-6

Figura 5. Sobrevivencia para plántulas establecidas con y sin FEC en un sitio seco, después de una temporada en terreno. Para todas las especies, la sobrevivencia de las plántulas fertilizadas fue significativamente menor en relación a las no fertilizadas (52).

Seedling survival after one field season. Planting with and without CRF on a dry site. For every species, survival of fertilized trees was significantly lower than survival of unfertilized seedlings. (52). 
BOSQUE 25(2): 89-100, 2004

RECOMENDACIONES. La mejor forma de utilizar los FEC es primero conocer los diversos tipos de productos y tecnologías disponibles y después probar en forma meticulosa una variedad de aplicaciones en diversas situaciones ambientales. Como hemos demostrado, no todos los FEC son similares, existiendo diferentes efectos bajo distintas situaciones de terreno. Pueden existir diversas fuentes y concentraciones de $\mathrm{N}$ en distintos fertilizantes. Por lo tanto, es responsabilidad del viverista o del encargado de la forestación entender claramente la tecnología y comparar la disponibilidad de productos bajo diversas situaciones específicas. El cuadro 1 enumera algunas recomendaciones para el uso de FEC.

DESAFIOS A LA INVESTIGACION. A pesar de que los FEC han sido utilizados por décadas en la horticultura, el desarrollo de metodologías para aplicaciones en la reforestación ha sido bastante lento. Considerando el potencial que tienen los FEC como una herramienta que mejora la situación nutricional de las plantas, es tiempo que los productores de plantas y forestadores coordinen esfuerzos para determinar las mejores tecnologías para distintos escenarios de plantación para una variedad de especies.

Algunos puntos que deben ser desarrollados y que merecen atención son los siguientes:

- Evaluar la interacción entre los FEC y otros factores como el clima, micrositio, competencia de malezas, especies, genética, etc., resultará en una herramienta fundamental para comprender el óptimo uso de los fertilizantes en terreno.

- Determinar mezclas de FEC y productos solubles para una óptima nutrición de las plantas, tanto en vivero como en terreno. A la fecha, se han realizado pocos estudios que exploren esta posibilidad, siendo que la mayoría de los productores han desarrollado sus propias mezclas con varias combinaciones adaptadas a sus condiciones locales.

- Otro objetivo importante es lograr ajustar la entrega de nutrientes a los objetivos de manejo. Por ejemplo, en aplicaciones en vivero, sería ventajoso aplicar FEC al momento de la siembra, pero sin liberación de nutrientes hasta por lo menos 12 ó 14 meses después del mo- mento de la plantación. Otra estrategia de manejo podría ser incorporar un FEC de tres meses que trabaje hasta la etapa de endurecimiento de las plantas, acompañado de otro fertilizante que libere nutrientes para la etapa de desarrollo radicular durante el otoño.

- Determinar la mejor combinación y aplicación de acuerdo a la especie y su estado de desarrollo. Diversas especies responden de manera distinta a las aplicaciones de nutrientes y otras especies son más tolerantes a la concentración de sales.

- Determinar distintas formulaciones y tasas de aplicación de acuerdo a condiciones de humedad de suelo resulta crucial al momento de enfrentar el estudio de estos fertilizantes. Estudios que profundicen en las ventajas asociadas a productos con $\mathrm{N}$ de lenta solubilidad en agua, en donde la liberación está estrechamente relacionada con el contenido de humedad del suelo, tienen un gran potencial para mejorar las recomendaciones de fertilizantes en sitios secos.

- Existen una serie de sistemas de aplicación: superficial, cercano a las raíces o en el mismo hoyo de plantación. La proximidad al sistema radicular resulta de vital importancia para la aplicación de nutrientes con poca movilidad. A diferencia de los fertilizantes solubles, el sistema de entrega prolongada y más lento de nutrientes puede aliviar problemas de toxicidad cuando los fertilizantes son aplicados próximos a las raíces. Sin embargo, aplicaciones cercanas al sistema radicular pueden causar daños por toxicidad en sitios secos. Recomendaciones sitioespecificas necesitan ser desarrolladas y evaluadas.

- La mayoría de las aplicaciones de FEC se realizan solamente una vez. Sin embargo, existe un creciente interés en realizar múltiples aplicaciones para estimular el crecimiento en terreno. Aplicaciones posteriores podrían aumentar los niveles de crecimiento y, por consiguiente, acortar los períodos de rotación.

En resumen, vemos una excelente oportunidad en relación al uso de FEC. Existen un sinnúmero de especies y productos disponibles para ser pro- 
bados en viveros y en el lugar de plantación. En la medida que la industria de FEC continúe mejorando la disponibilidad de productos, podremos esperar un creciente interés en relación a la forma en que estas tecnologías trabajan y como mejoran los resultados en viveros y en terreno.

\section{CUADRO 1}

Recomendaciones para el uso de fertilizantes de entrega controlada en viveros y en operaciones de plantación.

- Nunca aplicar un FEC, en particular a escala, sin haber primero experimentado. No existen dos situaciones de vivero o terreno iguales. Por lo tanto, resulta obligatorio experimentar a pequeña escala.

- Ser cuidadoso en interpretar y extrapolar información de artículos y opiniones de otras aplicaciones. Las condiciones de suelo pueden ser extremadamente diferentes.

- Asegurarse de que el producto es nuevo. A menos de que se posea un buen sistema de almacenamiento en bolsas totalmente aisladas, los fertilizantes absorben agua con el tiempo, endureciéndose o liberando nutrientes.

- Obtener toda la información de los fertilizantes y ser capaz de comprender en profundidad su composición. Usted debería saber las cantidades de N, P y K, Ca, Mg y B, como también la solubilidad y el tiempo de entrega de los elementos principales. Pregunte sobre los sistemas de recubrimiento y cómo funcionan. Las perlas deben ser uniformes en tamaño o se desarmarán con facilidad.

- Investigue la adaptabilidad de un producto en particular a su cultivo. Una combinación específica de nutrientes y sistemas de cubiertas de fertilizantes puede ser mejor para su situación en particular.

- La oportunidad en que se hace todo es la clave. Asegúrese de que las características de liberación coincidan con el período de crecimiento. En el vivero es necesario trabajar con el correcto sistema de irrigación y monitorear la conductividad eléctrica para evitar la toxicidad de sales.

- Manipular las bolsas de fertilizantes en forma cuidadosa, pues pueden sufrir de abrasión.

\section{BIBLIOGRAFIA}

(1) STROTHMANN. Large stock and fertilizer improve growth of Douglas-fir planted on unstable granitic soil in northern California. USDA Forest Service. 1980. Res. Note PSW-345.

(2) CARLSON, W.C., C.L. PREISIG. Effects of controlledrelease fertilizers on the shoot and root development of Douglas-fir seedlings. Can. J. For. Res. 1981, vol. 11, p. 230-242.

(3) CARLSON, W.C. Effects of controlled-release fertilizers on the shoot and root development of outplanted western hemlock (Tsuga heterophylla Raf. Sarg.) seedlings. Can. J. For. Res. 1981, vol. 11, p. 752-757.

(4) KRASOWSKI, M.J., J.N. OWENS, L.E. TACKABERRY, H.B. MASSICOTTE, A. STOKES. Above- and belowground growth of white spruce seedlings with roots divided into different substrates with or without controlled-release fertilizer. Plant Soil. 1999, vol. 11, p. 131-143.

(5) ROSE, R., D.L. HAASE, D. BOYER. Organic matter management in forest tree nurseries: theory and practice. Oregon State University, Nursery Technology Cooperative; Corvallis; USA. 1995. 67 p.

(6) MCNAB, W.H., C.R. BERRY. Distribution of aboveground biomass in three pine species planted on a devastated site amended with sewage sludge or inorganic fertilizer. For. Sci. 1985, vol. 31, p. 373-382.

(7) HARRISON, R.B., C.L. HENRY, D.S. XUE. Magnesium deficiency in Douglas-fir and grand fir growing on a sandy outwash soil amended with sewage sludge. Water Air Soil Poll. 1994, vol. 75, p. 37-50.

(8) WEETMAN, G.F., M.A. MCDONALD, C.E. PRESCOTT, J.P. KIMMINS. Responses of western hemlock, Pacific silver fir, and western red cedar plantations on northern Vancouver Island to applications of sewage sludge and inorganic fertilizer. Can. J. For. Res. 1993, vol. 23, p. 1815-1820.

(9) PRESCOTT, C.E., L.M. ZABEK. Growth response and nutrient availability in western redcedar plantations following amendment with fish-wood compost and straw. Can. J. For. Res. 1997, vol. 27, p. 598-602.

(10) GOERTZ, H.M. Controlled Release Technology, p. 251274. In: Kirk-Othmer Encyclopedia of Chemical Technology, vol. 7, $4^{\text {th }}$ ed. Wiley, New York. 1993.

(11) EWEN, H., T. JAHNS. Bacterial degradation of methyleneureas used as slow-release fertilizer. Recent Research Developments in Microbiology. 2000, vol. 4, p. 537-546.

(12) AUSTIN, R.C., R.F. STRAND. The use of slowly soluble fertilizers in forest planting in the Pacific Northwest. J. For. 1960, vol. 58, p. 619-627.

(13) AARNIO, T., P.J. MARTIKAINEN. Mineralization of carbon and nitrogen, and nitrification in Scots pine forest soil treated with fast- and slow-release nitrogen fertilizers. Biol. Fertil. Soils. Berlin, Germany: Springer-Verlag. 1996, vol. 22, p. 214-220.

(14) GLEASON, J.F., M. DURYEA, R. ROSE, M. ATKINSON. Nursery and field fertilization of $2+0$ ponderosa pine seedlings: the effect on morphology, physiology, and field performance. Can. J. For. Res. 1990, vol. 20, p. 1766-1772.

(15) KHATAMIAN, H., J.C. PAIR, R. CARROW. Effects of turf competition and fertilizer application on trunk diameter and nutrient composition of honeylocust. J. Arbor. 1984, vol. 10, p. 156-159.

(16) ARELLANO, E. The effect of soil type, fertilizer, and moisture regime on $1+0$ Douglas fir seedlings. M.Sc. thesis, Oregon State Univ. Corvallis. USA. 2003. 88 p. 
(17) HAUCK, R.D. Slow-release and bioinhibitor-amended nitrogen fertilizers. P. 293-322. In: Fertilizer Technology and Use, $3^{\text {rd }}$ ed., Soil Science Society of America, Inc., Madison, WI. 1985.

(18) STRUVE, D.K. Nitrogen, phosphorus and potassium recovery of container-grown red oak and blackgum seedlings under different fertilizer application methods. $J$. Env. Hort. 1995, vol. 13, p. 169-175.

(19) ROSE, R., J.S. KETCHUM. Interaction of vegetation control and fertilization on conifer species across the Pacific Northwest. Can. J. For. Res. 2002, vol. 32, p. 136152.

(20) NAMBIAR, E.K.S., K.M. CELLIER. Nitrogen fertilisers in establishing Pinus radiata plantations on sandy soils: an evaluation of their use. Aust. For. 1985, vol. 48, p. 242251.

(21) BENSON, R.B. Fertilizer Technology. p. 5-9 in Proc. of Symposium on Forest Seedling Nutrition from the Nursery to the Field, OSU College of Forestry, October 28-29, Corvallis, OR. 1997.

(22) RADWAN, M.A., D.S. DEBELL. Effects of different urea fertilizers on soil and trees in a young thinned stand of western hemlock. Soil Sci. Soc. Am. J. 1989, vol. 53, p. 941-946.

(23) LAMONT, G.P., R. J. WORRALL, M.A. O'CONNELL. The effects of temperature and time on the solubility of resin-coated controlled-release fertilizers under laboratory and field conditions. Sci. Hort. 1987, vol. 32, p. 265-273.

(24) HICKLENTON, P.R., K.G. CAIRNS. Solubility and application rate of controlled-release fertilizer affect growth and nutrient uptake in containerized woody landscape plants. J. of the Amer. Soc. for Hort. Sci. 1992, vol. 117, p. 578583.

(25) GAMBASH, S., M. KOCHBA, Y. AVNIMELECH. Studies on slow-release fertilizers: II. A method for evaluation of nutrient release rate from slow-releasing fertilizers. Soil Sci. 1990, vol. 150, p. 446-450.

(26) KOCHBA, M., S. GAMBASH, Y. AVNIMELECH. Studies on slow release fertilizers. 1. Effects of temperature, moisture, and water vapour pressure. Soil Sci. 1990, vol. 149, p. 339-343.

(27) HUETT, D.O., S.C. MORRIS. Fertiliser use efficiency by containerised nursery plants - 3. Effect of heavy leaching and damaged fertiliser prills on plant growth, nutrient uptake, and nutrient loss. Aust. J. Agric. Res. 1999, vol. 50, p. 217-222.

(28) GROVES, K.M., S.L. WARREN, T.E. BILDERBACK. Irrigation volume, application, and controlled-release fertilizers: I. Effect on plant growth and mineral nutrient content in containerized plant production. J. Environ. Hort. 1998, vol. 16, p. 176-181.

(29) ARNOTT, J.T., A.N. BURDETT. Early growth of planted western hemlock in relation to stock type and controlledrelease fertilizer application. Can. J. For. Res. 1988, vol. 18, p. 710-717.

(30) REDDELL, P., M.J. WEBB, D. POA, D. AIHUNA. Incorporation of slow-release fertilisers into nursery media. New For. 1999, vol. 18, p. 277-287.

(31) BRAND, D.G., P.S. JANAS. Growth and acclimation of planted white pine and white spruce seedlings in response to environmental conditions. Can. J. For. Res. 1988, vol. 18 , p. $320-329$

(32) TOWNSEND, J. Effects of elevated $\mathrm{CO}_{2}$, water and nutrients on Picea sitchensis (Bong.) Carr. seedlings. New Phytol. 1995, vol. 130, p. 193-206.

(33) WALKER, R.F. Artificial regeneration of Jeffrey pine in the Sierra Nevada: growth, nutrition, and water relations as influenced by controlled release fertilization and solar protection. J. Sustain. For. 1999, vol. 9, p. 23-38.

(34) HUNT, G.A. Effect of controlled-release fertilizers on growth and mycorrhizae in container-grown Engelmann spruce. West. J. Appl. For. 1989, vol. 4, p. 129-131.

(35) WALKER, R.F., C.D. HUNTT. Controlled release fertilizer effects on growth and foliar nutrient concentration of container grown Jeffrey pine and singleleaf pinyon. West. J. Appl. For. 1992, vol. 7, p. 113-117.

(36) CASTELlANO, M.A., J.M. TRAPPE, R. MOLINA Inoculation of container-grown Douglas-fir seedlings with basidiospores of Rhizopogon vinicolor and R. colossus: effects of fertility and spore application rate. Can. J. For. Res. 1985, vol. 15, p. 10-13.

(37) CHAKRAVARTY, P., L. CHATARPAUL. Effect of fertilization on seedling growth, ectomycorrhizal symbiosis, and nutrient uptake in Larix laricina. Can. J. For. Res. 1990, vol. 20, p. 245-248.

(38) SHAW, C.G. III, R.M. JACKSON, G.W. THOMAS. Fertilizer levels and fungal strain influence the development of ectomycorrhizae on Sitka spruce seedlings. New For. 1987, vol. 1, p. 215-223.

(39) NURSERY TECHNOLOGY COOPERATIVE (NTC). Field performance of Douglas-fir container stock grown with fertilizer-amended media. p. 28-32 In: 2001-02 NTC Annual Report. Department of Forest Science, Oregon State University. 2002. 63 p.

(40) BROSCHAT, T.K. Release rates of soluble and controlledrelease potassium fertilizers. HortTech. 1996, vol. 6, p. $128-131$.

(41) WANG, F.L., A.K. ALVA. Leaching of nitrogen from slow-release urea sources in sandy soils. Soil Sci. Soc. Am. J. 1996, vol. 60, p. 1454-1458.

(42) WALKER, R.F., C.D. HUNTT. Growth and nutrition of containerized singleleaf pinyon seedlings in response to controlled release fertilization. Arid Soil Res. Rehab. 1999, vol. $13, \mathrm{~N}^{\mathrm{o}} 2$, p. 123-132.

(43) WALKER, R.F. Y C.D. HUNTT. Production of containerized Jeffrey pine planting stock for harsh sites Growh and nutrition as influenced by controlled-release fertilization. West. J. Appl. For. 2000, vol. 15, p. 86-91.

(44) HUETT, D.O. Fertiliser use efficiency by containerized nursery plants. 1. Plant growth and nutrient uptake. Aust. J. Agric. Res. 1997, vol. 48, p. 251-258.

(45) HUETT, D.O., B.J. GOGEL. Longevities and nitrogen, phosphorus, and potassium release patterns of polymercoated controlled-release fertilizers at $30^{\circ} \mathrm{C}$ and $40^{\circ} \mathrm{C}$ Commun. Soil Sci. Plan. 2000, vol. 31, p. 959-973.

(46) CABRERA, R.I. Comparative evaluation of nitrogen release patterns from controlled-release fertilizers by nitrogen leaching analysis. Hort Sci. 1997, vol. 32, p. 669673.

(47) HANDRECK, K. Controlled release fertilisers. Measuring nutrient release rates. Aust. Hort. 1997, vol. 95, p. 51-53.

(48) ALZUGARAY, P. Effects of fertilization at the time of planting on field performance of $1+1$ Douglas-fir (Pseudotsuga menziesii (Mirb.) Franco) seedlings. M.Sc. thesis, Oregon State Univ. 2002, 128 p.

(49) POWERS, R.F., P.E. REYNOLDS. Ten-year responses of ponderosa pine plantations to repeated vegetation and nutrient control along an environmental gradient. Can. J. For. Res. 1999, vol. 29, p. 1027-1038.

(50) BROCKLEY, R.P. The effects of fertilization on the early growth of planted seedlings: a problem analysis. FRDA Report Victoria, B.C. 1988. № 011, 16 p. 
BOSQUE 25(2): 89-100, 2004

Fertilizantes de entrega controlada...

(51) SOUTH, D.B., J.B. ZWOLINSKI, H.L. ALLEN Economic returns from enhancing loblolly pine establishment on two upland sites: effects of seedling grade, fertilization, hexazinone, and intensive soil cultivation. New For. 1995, vol. 10, p. 239-256.
(52) NURSERY TECHNOLOGY COOPERATIVE (NTC). Fertilization with slow relase fertilizers on high and low elevation sites at the Warm Springs Indian Reservation. p. 20-22 In: 1998-99 NTC Annual Report. Department of Forest Science, Oregon State University. 1999, 40 p.

Recibido: 03.10 .03 